\title{
Influence of Reductant Concentration on the Conduction Path Formation Properties of Solderable Polymer Composites
}

\author{
Byung-Seung Yim ${ }^{1}$, Jeong Il Lee ${ }^{2}$ and Jong-Min Kim²,* \\ ${ }^{1}$ Department of Mechanical Engineering, Kangwon National University, Gangwon-do 25913, Korea \\ ${ }^{2}$ School of Mechanical Engineering, Chung-Ang University, Seoul 156-756, Korea
}

When solderable polymer composites (SPCs) filled with a low-melting-point-alloy (LMPA) was used in the bonding process, the elimination of surface oxide layers of the fillers and electrodes is very important to achieve an excellent conduction path by the proper coalescence-wetting behaviors of molten fillers. In this study, to investigate the influence of reductant content on the conduction path formation, four kinds of SPC with different reductant amounts $(0,7.5,15$, and $30 \mathrm{phr})$ were examined. The LMPA filler with a reductant concentration of $15 \mathrm{phr}$ exhibits excellent wettability because of the proper elimination of the surface oxide layer of fillers and electrodes. However, the wettability deteriorated with a further increased reductant content due to the rapid curing of the SPC at low temperature conditions caused by increased chemical reactions between the carboxyl groups of the reductant and epoxide groups. [doi:10.2320/matertrans.M2018067]

(Received February 16, 2018; Accepted May 28, 2018; Published July 23, 2018)

Keywords: electrically conductive adhesive (ECA), low-melting-point alloy, polymeric composites, reductant

\section{Introduction}

Conductive filler filled polymeric composites (i.e., electrically conductive adhesives (ECAs)) have been widely used for a wide range of applications as a potential substitute for solder materials used in the microelectronics industry. These polymeric composites have very versatile properties, which include high thermal stability, low electrical resistance, favorable mechanical response and adhesive characteristics. $^{1,2)}$ For health and environmental reasons alone, these composites have attracted increasing attention due to their capability for more flexible and simpler processing at a lower temperature, combined with the capability to bond non-solderable materials. ${ }^{3-5)}$ However, in spite of these advantages, complete replacement of soldering processes by ECAs is not yet possible because of several critical limitations, such as low electrical and thermal conductivity, unstable contact resistance with nonnoble metal finished components, and poor mechanical performance compared with traditional solder material. ${ }^{6,7)}$

In our previous study, low-melting-point-alloy (LMPA) filled solderable polymer composites (SPCs) were introduced, and their electrical and mechanical properties and thermomechanical reliability properties were investigated. ${ }^{8-10)}$ The SPCs have an inherent reduction capability represented by $\mathrm{R}(\mathrm{COOH})_{\mathrm{x}}$ ( $\mathrm{x}$ is greater than or equal 2$)$. During the reflow process, the surface oxide layers of the fillers and electrodes on the substrate are eliminated by reduction of the SPC according to the following chemical reaction:

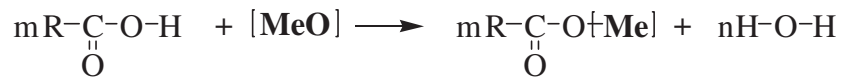

As shown in Fig. 1, the electrical conduction path is mainly established by rheology-coalescence-wetting behaviors of the molten LMPA fillers in the SPC during the reflow process. If the surface oxide layers of fillers and electrodes are poorly eliminated, the flow-coalescence-wetting behaviors of LMPA fillers may be hindered. Therefore, the surface oxide layers

*Corresponding author, E-mail: 0326kjm@cau.ac.kr

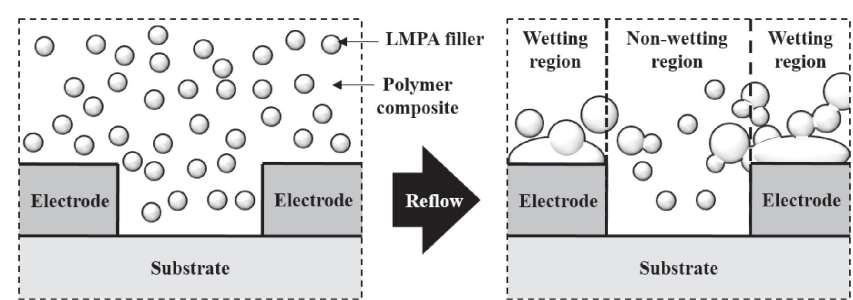

Fig. 1 Schematic of the coalescence and wetting behaviors of the molten LMPA filler in the formulated SPC with reduction capability.

of fillers and electrodes should be removed completely by the reduction capability of the SPC; thus, the reductant concentration in an SPC is a very important factor to achieve excellent conduction path formation using SPCs.

The goal of this study is to investigate the influence of reductant concentration on the coalescence and wetting behaviors of LMPAs in the SPCs. Chip resistor bonding was conducted using the formulated SPCs, and the morphologies of the conduction paths with different reductant loadings were observed and compared. In addition, the mechanical properties of the resulting assemblies were investigated.

\section{Experimental}

The developed SPCs consist of an LMPA, a thermoset epoxy resin, and other minor organic additives. A diglycidyl ether of bisphenol A (DGEBA; Kukdo Chemical; epoxy equivalent weight $($ E.E.W. $)=186.8 \mathrm{~g} / \mathrm{eq}$ ) was used as the binder, and 4,4'-diaminodiphenylmethane (DDM; TCI Korea) and boron trifluoro-mono-ethylamine $\left(\mathrm{BF}_{3} \mathrm{MEA}\right.$; Wako Pure Chemical) were used as the curing agent and catalyst, respectively. 3-Butanoic acid (Aldrich; 97\%) was used as a reductant to remove the oxide layer on the surfaces of the LMPA and the electrode. The LMPA solder powder $\left(\mathrm{Sn}-58 \mathrm{Bi} ; \mathrm{T}_{\mathrm{m}}=412 \mathrm{~K} ; 45 \mu \mathrm{m}\right)$ was obtained from Duksan Hi-Metal Co. To compare the coalescence and wetting behaviors of the LMPA fillers within the formulated SPCs, four kinds of SPC with $40 \mathrm{vol} \%$ LMPA and different 
Table 1 Components of the SPC formulations used.

\begin{tabular}{cll}
\hline \multirow{4}{*}{ Polymer Composite } & \multicolumn{2}{c}{ Components } \\
\cline { 2 - 3 } & \multicolumn{1}{c}{ Base Resin } & DGEBA \\
\cline { 2 - 3 } & Curing Agent & DDM \\
\cline { 2 - 3 } & Catalyst & $\mathrm{BF}_{3}$ MEA \\
\cline { 2 - 3 } Leductant & Carboxylic Acid: $0,7.5,15$ and $30 \mathrm{phr}$ \\
\hline \multirow{2}{*}{ LMPA Filler } & Composition & $\mathrm{Sn} / 58 \mathrm{Bi}$ \\
\cline { 2 - 3 } & Diameter $(\mu \mathrm{m})$ & 45 \\
\cline { 2 - 3 } & Content $(\mathrm{vol} \%)$ & 40 \\
\hline
\end{tabular}

reductant concentrations $(0,7.5,15$, and $30 \mathrm{phr})$ were formulated. The SPC formulations are shown in Table 1.

For the adhesive bonding test, a 104-chip resistor with I/O terminals plated with Sn (Samsung Electro-Mechanics Co. Ltd.; $6.30 \times 3.20 \times 0.60 \mathrm{~mm}^{3}$ in size) and a Cu-plated printed circuit board (PCB; $32 \times 32 \times 1.0 \mathrm{~mm}^{3}$ in size) were used. The substrate was cleaned with acetone for $1 \mathrm{~min}$, rinsed with DI water, and dried with an air jet. Formulated SPCs with 40 vol\% LMPA fillers were selectively applied on the conducting pads by the squeegee method. The chip resistor was then aligned and mounted on the $\mathrm{PCB}$, and adhesive bonding was conducted using chip bonder (LAMBDA; FINETECH Co.). In the adhesive bonding process, the test vehicle was heated from room temperature to $433 \mathrm{~K}$ at a heating rate of $10 \mathrm{~K} / \mathrm{min}$ and maintained for $20 \mathrm{~s}$, and then it was heated to $453 \mathrm{~K}$ and maintained for $80 \mathrm{~s}$ to complete curing of SPCs. After the adhesive bonding, the morphology of the conduction path was observed using an optical microscope (VHX-1000; KEYENCE Co.).

To observe the mechanical property of each SPC joint with different reductant concentrations, a shear test was performed using a shear testing machine (PTR-1000; Rhesca Co.) for the five test samples per different reductant concentration. The shear jig was put in contact with the chip resistor, which was then sheared in the parallel direction to the substrate with a shear speed of $10 \mathrm{~mm} / \mathrm{min}$, according to the standard JIS Z 3198-7. Additionally, to investigate the effect of reductant concentration on the chemical reactions of the epoxy system, differential scanning calorimetry (DSC; DSC 131 Evo; SETARAM Instrument) analysis was conducted for the mixture of epoxy and reductant. A mixture of 8$12 \mathrm{mg}$ was put into a DSC pan in an air-purged nitrogen environment. The change in heat flow was then measured during heating from room temperature to $573 \mathrm{~K}$ at a heating rate of $10 \mathrm{~K} / \mathrm{min}$.

\section{Results and Discussion}

Figure 2 shows the morphology of the conduction path of each formulated SPC with different reductant concentrations. The interconnected parts were divided into two distinct regions. One is a conductive path made by melted LMPA fillers, and the other is a cured polymer wrapped around the outside of the conduction path. The white dotted line indicates the polymer region. As can be seen in Fig. 2(a), the LMPA fillers in the SPC with $0 \mathrm{phr}$ reductant are neither coalesced nor wetted. Although the LMPA fillers are in physical contact, the coalescence between the fillers is not remarkable, and most fillers remain in their original condition because the remaining oxide on the surfaces of filler particles and electrodes hinders the coalescence and wetting behaviors. Meanwhile, as shown in the Figs. 2(b) and (c), the wettability of LMPA fillers increasingly improves as the amount of reductant is increased; simultaneously, the amount of remaining fillers decreases, and the conduction path area
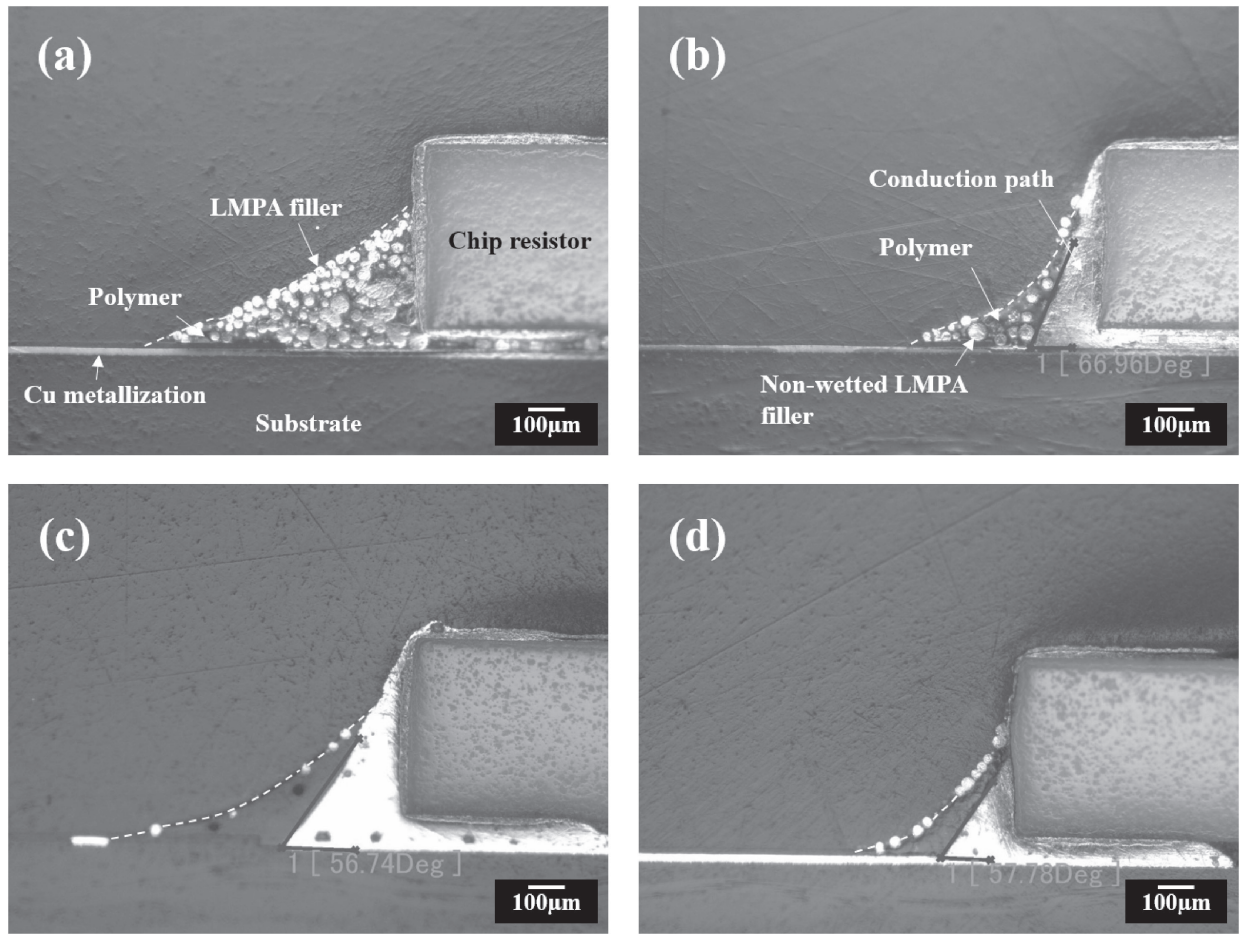

Fig. 2 Morphology of the conduction path formulated between metallizations of the chip resistors and PCB substrates by SPCs with reductant concentrations of (a) 0 , (b) 7.5 , (c) 15 , and (d) $30 \mathrm{phr}$. 


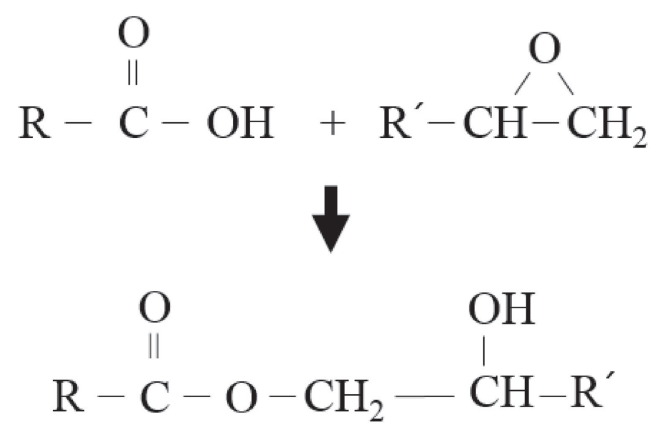

Fig. 3 Chemical reaction between the carboxyl group of the reductant and the epoxide group of the polymer composite. $\mathrm{R}$ and $\mathrm{R}^{\prime}$ are organic.

increases with increasing reductant content. This phenomenon is attributed to improvement of the reduction capabilities of the SPC. However, as shown in Fig. 2(d), the wettability of LMPA fillers becomes poor as the concentration of the reductant is increased over a concentration of $15 \mathrm{phr}$; the amount of non-wetted residual LMPA filler increased within the cured polymer region, and the conduction path area decreased with increasing reductant content. The LMPA filler with a reductant concentration of $15 \mathrm{phr}$ exhibits excellent wettability and the widest conduction path area compared with both 7.5 and $30 \mathrm{phr}$ reductant concentrations.

Such a phenomenon (i.e., decreased wettability of fillers depending on increased reductant concentration) may be attributed to the chemical reaction between the carboxyl groups of the reductant and the epoxide groups of the SPC. As shown in Fig. 3, the carboxyl groups of the reductant participate in the opening of epoxide rings, resulting in ester bonds and formation of hydroxyl $(-\mathrm{OH})$ groups. ${ }^{11,12)}$ This reaction between the carboxyl groups and epoxide groups has an impact on both the curing rate and viscosity of the SPCs. Therefore, a rapid curing of the SPC at low temperature conditions due to increased active chemical reactivity with the increased reductant content prevents the proper flow, coalescence, and wetting behaviors of the molten LMPA fillers within the SPC.

To validate the chemical reaction in Fig. 3, DSC analysis was conducted for the mixture of epoxy and reductant, as shown in Fig. 4. As can be seen in the results, the pure epoxy (without any additives) did not show any heat flow change over the entire temperature range. Meanwhile, the mixture with a reductant concentration of $7.5 \mathrm{phr}$ showed an exothermic peak by curing at $474.49 \mathrm{~K}$, and the exothermic peak temperature shifted toward a lower temperature with an increase in reductant content (exothermic peak temperature: $460.84 \mathrm{~K}$ for epoxy with a reductant concentration of $15 \mathrm{phr}$ and $434.53 \mathrm{~K}$ for epoxy with reductant concentration of $30 \mathrm{phr}$ ). The DSC analysis results showed that the carboxyl groups of the reductant react with the epoxide groups. Due to these reactions, with an increasing reductant concentration, the curing reaction of the SPC occurred rapidly at low temperature range during the reflow process because of increased chemical reactivity (i.e., curing). Such a rapidly occurred curing reaction of the SPC hinders the fluidity of the molten LMPA, the coalescence behaviors between adjacent molten fillers by contact, and the wetting behavior between molten fillers and metallizations.

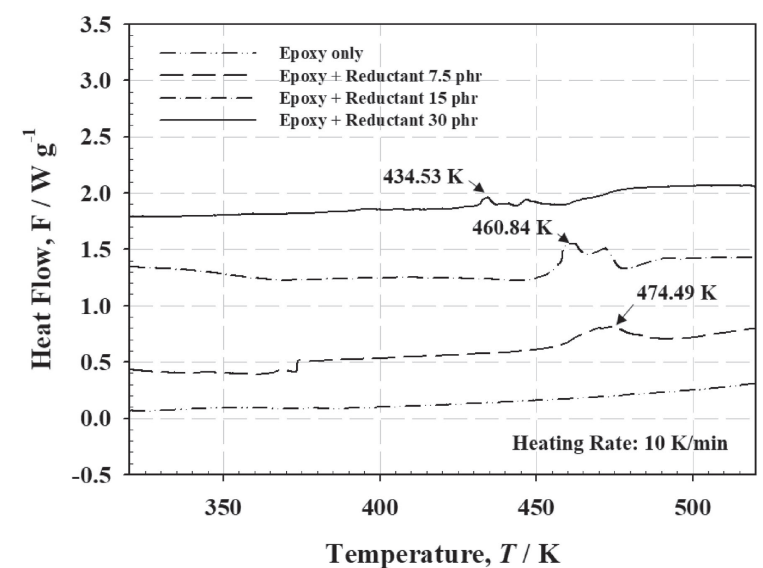

Fig. 4 Dynamic DSC analysis results for the epoxy with different reductant concentrations.

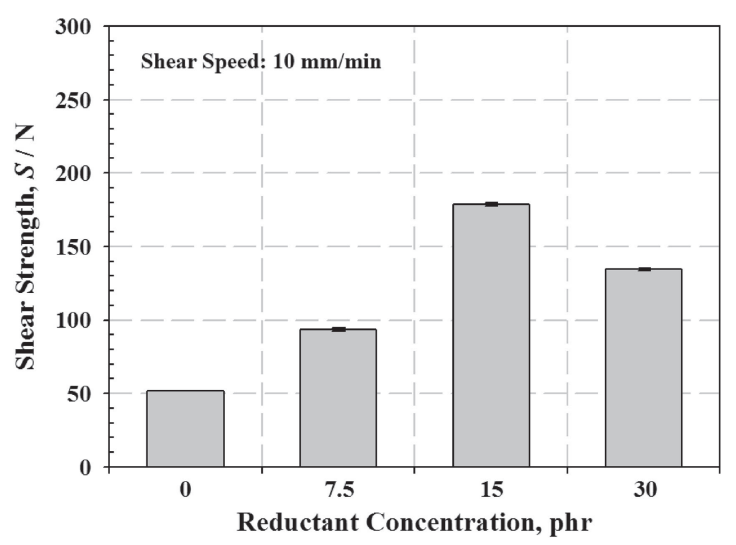

Fig. 5 Shear strengths of chip resistor joints for SPCs with different reductant concentrations.

Figure 5 shows the shear test results for the chip resistor assemblies using SPCs with different reductant concentrations. The shear strength of the chip resistor assembly joint is directly affected by the conduction path area. As shown in Fig. 2, the LMPA filler with a reductant concentration of $15 \mathrm{phr}$ exhibits the widest conduction path area compared to both 7.5 and $30 \mathrm{phr}$ reductant concentrations, because of the excellent wettability. As can be seen in the shear test results, the chip resistor assemblies using SPC with a $15 \mathrm{phr}$ reductant concentration exhibited improved shear strength $(178.71 \mathrm{~N})$ compared to those of the joints using SPC with reductant concentrations of 0 and $7.5 \mathrm{phr}(51.84 \mathrm{~N}$ for reductant $0 \mathrm{phr}$ and $93.48 \mathrm{~N}$ for reductant $7.5 \mathrm{phr}$ ). However, the mechanical properties deteriorated with a further increased reductant content $(134.39 \mathrm{~N}$ for a reductant concentration of $30 \mathrm{phr}$ ) because of the decreasing conduction path area.

\section{Conclusion}

In this study, an SPC with LMPA was fabricated, and the influence of reductant concentration on the conduction path formation properties of the SPC was investigated. The chip resistor bonding test results showed that the chip resistor joint using an SPC without reductant showed a weak conduction path formation due to the absence of reduction capability. Meanwhile, when the reduction capability was introduced 
into the SPCs, a stable conductive path with a large area was formed due to improved removal of the surface oxide film as the concentration of the reductant increased, and the mechanical shear strength also increased. However, the wettability of molten LMPA deteriorated with a further increased reductant concentration $(30 \mathrm{phr})$ due to the rapid curing of the SPC at low temperature conditions caused by increased chemical reactions between the carboxyl groups of the reductant and the epoxide groups.

From the results of the present study, we conclude that an optimized reductant concentration in the formulated SPCs acts as an important factor to achieve the most favorable interconnection joints.

\section{Acknowledgements}

This research was supported by Basic Science Research Program through the National Research Foundation of Korea (NRF) funded by the Ministry of Science, ICT and future Planning (Grant No. 2017R1C1B5076997) and the ChungAng University Research Scholarship Grants in 2016.

\section{REFERENCES}

1) M.J. Yim, Y. Li, K.S. Moon, K.W. Paik and C.P. Wong: J. Adhes. Sci. Technol. 22 (2008) 1593-1630.

2) L.J. Matienzo, R.N. Das and F.D. Egitto: J. Adhes. Sci. Technol. 22 (2008) 853-869.

3) H.K. Kim and F.G. Shi: Microelectronics J. 32 (2001) 315-321.

4) M.J. Yim and K.W. Paik: Int. J. Adhes. Adhes. 26 (2006) 304-313.

5) X. Nana, L. Zhiling, X. Hui, Z. Yuzhen, W. Yuehui and L. Jingze: Rare Met. Mater. Eng. 45 (2016) 2524-2528.

6) H.W. Cui, D.S. Li, Q. Fan and H.X. Lai: Int. J. Adhes. Adhes. 44 (2013) 232-236.

7) S. Xu, D.A. Dillard and J.G. Dillard: Int. J. Adhes. Adhes. 23 (2003) 235-250.

8) B.S. Yim, J.M. Kim, S.H. Jeon, S.H. Lee, J. Kim, J.G. Han and M. Cho: Mater. Trans. 50 (2009) 2649-2655.

9) B.S. Yim, Y. Kwon, S.H. Oh, J. Kim, Y.E. Shi, S.H. Lee and J.M. Kim: Microelectron. Reliab. 52 (2012) 1165-1173.

10) B.S. Yim, J.I. Lee, Y. Heo, J. Kim, S.H. Lee, Y.E. Shin and J.M. Kim: Mater. Trans. 53 (2012) 2104-2110.

11) G. Tillet, B. Boutevin and B. Ameduri: Prog. Polym. Sci. 36 (2011) 191-217.

12) M. Abdalla, D. Dean, D. Adibempe, E. Nyairo, P. Robinson and G. Thompson: Polymer 48 (2007) 5662-5670. 